\title{
Nachweis von Adrenalin und Noradrenalin in kleinen Blutvolumina
}

\author{
Von D. Endel und G. Hollmann
}

Aus der kindercbirurgischen Abteilung (Vorstand: Prof. Dr. W. Ch. Hecker) der Chirurg. Univ.-Klinik Heidelberg

(Eingegangen am 26. Juni 1969)

Zur getrennten fluorometrischen Bestimmung von Adrenalin und Noradrenalin wird eine vereinfachte Nachweismethode beschrieben, die bei erhöhtem Katecholaminspiegel nur kleine Blutvolumina benötigt. Die Isolierung der Katecholamine aus dem Plasma erfolgt durch einen Kationenaustauscher in einer klein dimensionierten Säule. Durch Anwendung von Druck wird dieses Verfahren beschleunigt. Die Elution wird durch einmaliges Eichen der Säulen vereinfacht. Die Nachweisreaktion erfolgt nach der Trihydroxyindol-Methode. Durch Messung der Fluoreszenz bei zwei Anregungsmaxima und dem Vergleich mit bekannten Eichwerten werden Adrenalin und Noradrenalin quantitativ differenziert.

\section{Determination of adrenaline and noradrenaline in small blood volumes}

A simplified method is reported for the separate fuorometric determination of adrenaline and noradrenaline. In cases with increased levels of catecholamines, only small volumes of blood are required. Catecholamines are isolated from the plasma with a small dimension column of a cation exchanger, pressurised to increase the flow rate. The elution is simplified by a single precalibration of the column. The trihydroxyindole reaction is used for detection. Adrenalin and noradrenaline are differentiated quantitatively by measurement of the fluorescence at two excitation maxima and comparison with known standards.

Neben einem großen methodischen Aufwand erfordert die fluorometrische Bestimmung von Adrenalin und Noradrenalin Blutvolumina von 18 bis $50 \mathrm{ml}$ (1). Bei erhöhtem Katecholaminspiegel, z. B. in der Vena cava inferior oder im Streß, kann durch die folgende Modifikation der Methoden von BertLer (2) und HäGGENDAL (3) das Blutvolumen auf 1 bis $4 \mathrm{ml}$ reduziert und der Arbeitsablauf wesentlich beschleunigt und vereinfacht werden. Es wird dabei eine klein dimenșionierte Säule mit einem Kationenaustauscher kleinster Korngröße verwendet. Ein weiterer Vorteil dieser Säulen liegt in der Einsparung von Reagenzien und der Verringerung des Eluatvolumens. Durch die kleinen Blutvolumina eignet sich diese Modifikation für Verlaufskontrollen bei Kindern und Laboratoriumstieren.

\section{Methodik}

\section{Zubebör und Reagenzien}

1. Spektralfluorometer mit 2 Monochromatoren, Fa. Zeiss.

2. Pasteurpipetten, Länge $215 \mathrm{~mm}$, oberer Durchmesser 0,6 mm.

3. Rekordspritzen mit Luer-Lock-Ansatz, $5 \mathrm{~m} /$.

4. Kunststoffschlauch zur Adaptation von Spritze und Pasteurpipette.

5. Graduierte Kunststoffbecher, Volumen etwa $1 \mathrm{~m} /$.

6. Kationenaustauscher AG 50W-X8, minus 400 mesh, Fa. Bio-Rad.

7. Quartdest. Wasser.

8. $\mathrm{HCl}, 0,005 \mathrm{~N}, 1 \mathrm{~N}, 2 \mathrm{~N}$.

9. $\mathrm{NaOH}, 2 \mathrm{~N}+1 \%$ EDTA (Titriplex II), $10 \mathrm{~N}$.

10. Perchlorsäure, $4 \mathrm{~N}$.

11. Phosphatpuffer, $0,1 \mathrm{M}, \mathrm{pH} 6,5+0,1 \%$ EDTA (Titriplex $\Pi$ ).

12. $\mathrm{K}_{2} \mathrm{CO}_{3}, 5 \mathrm{~N}$.

13. $\mathrm{K}_{3} \mathrm{Fe}(\mathrm{CN})_{6}, 0,25$ proz.

14. $\mathrm{Na}_{2} \mathrm{SO}_{3} \cdot 7 \mathrm{H}_{2} \mathrm{O}, 5 \mathrm{~g}$ in $10 \mathrm{~m} / \mathrm{H}_{2} \mathrm{O}$ gelöst, 6 Tage haltbar.

15. BAL- $\mathrm{Na}_{2} \mathrm{SO}_{3}$-Lösung, $0,05 \mathrm{~m} / \mathrm{BAL}$ zu $10 \mathrm{~m} / \mathrm{Na}_{2} \mathrm{SO}_{3}$-Lösung, 2 Stdn. haltbar; BAL = 2,3-Dimercaptopropanol-1, Fa. Merck.

16. L-Adrenalinhydrogen-L-tartrat, Fa. Schuchardt.

17. L-Noradrenalintartrat, $\mathrm{Fa}$. Serva.

\section{Prinzip des Nachweises}

Die Isolierung der Katecholamine aus dem Serum erfolgt durch einen stark sauren Kationenaustauscher. Durch ein Oxydationsmittel werden im Eluat Adrenalin und Noradrenalin zu Adreno- und Noradrenochrom oxydiert (4). Im alkalischen Milieu bilden sich die stark fluoreszierenden Trihydroxyindole Adreno- und Noradrenolutin $(5,6)$. Die unterschiedlich hohen Emissionen bei verschiedenen Anregungsmaxima ermöglichen die quantitative Differenzierung $(7,8)$.

\section{Blutentnabme}

1 bis $4 \mathrm{~m} l$ zentralvenöses Blut wird sofort nach Entnahme in eisgekühlten Plastikröhrchen 3 bis 4 Min. bei 15000 U./Min. (27750 g) zentrifugiert. Die abpipettierte und gemessene Serummenge wird mit $1 / 10$ ihres Volumens Perchlorsäure enteiweißt und nach 5 Min. Kühlung kurz zentrifugiert. Der klare Überstand wird abgegossen und eingefroren. Schnelle Kühlung und Verarbeitung des entnommenen Blutes sind notwendig, um den Verlust an Katecholaminen klein zu halten.

\section{Herstellung der Säulen}

Das ausgezogene Ende der Pasteurpipetten wird auf $2 \mathrm{~cm}$ gekürzt, die Öffnung durch Zuschmelzen etwas verkleinert und mit Glaswolle nicht zu fest verstopft. 40 bis $70 \mathrm{mg}$ Austauscherharz werden mit Wasser blasenfrei eingefültt. Die erforderliche Austauscherharzmenge richtet sich nach dem Feuchtigkeitsgehalt und wird durch einmaliges Eichen der Säulen bestimmt (siehe unten).

\section{Waschen der Säulen}

Der Austauscher muß durch Waschen von Rückständen befreit und in die beste Reaktionslage gebracht 


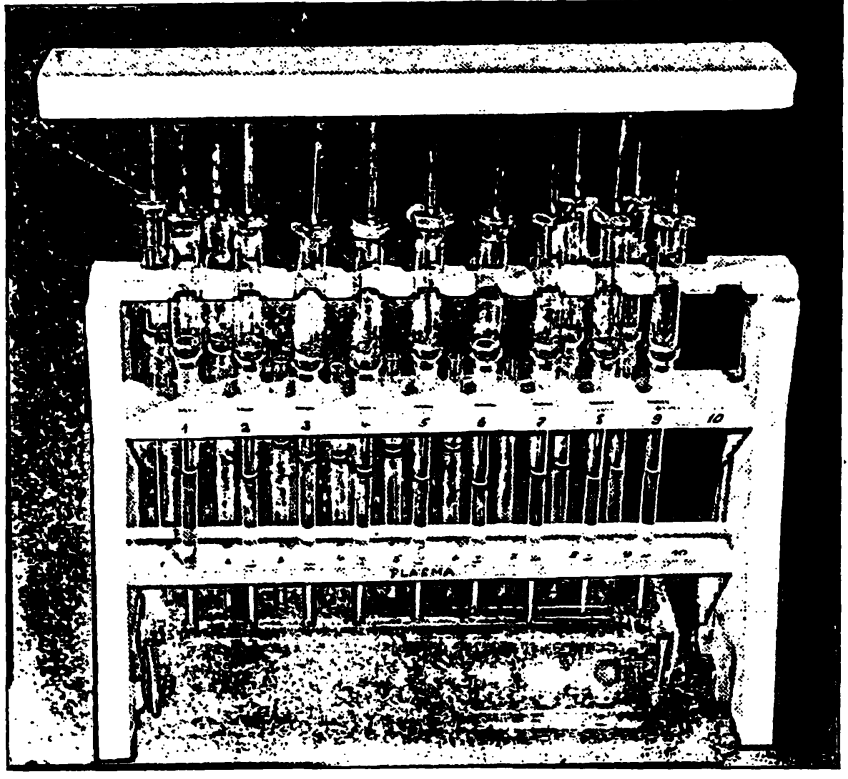

Abb. 1

Waschen der Säulen. Durch geeignete Anordnung können bis zu 20 Säulen gleichzeitig gewaschen werden

werden. Unter Druck werden folgende Spülflüssigkeiten nacheinander durch die Säule gepreßt:

$$
\begin{aligned}
& 3 \mathrm{~m} l 2 \mathrm{~N} \mathrm{NaOH} \\
& 3 \mathrm{~m} l \text { quartdest. Wasser } \\
& 3 \mathrm{~m} l 2 \mathrm{~N} \mathrm{HCl} \\
& 3 \mathrm{~m} l \text { quartdest. Wasser } \\
& 3 \mathrm{~m} l \text { Phosphatpuffer } \\
& 1 \mathrm{~m} l \text { quartdest. Wasser. }
\end{aligned}
$$

Die Durchflußgeschwindigkeit wird mit Hilfe von Rekordspritzen erhöht, deren Konus mit einer Kunststoffmuffe luftdicht auf die Säulen aufgesetzt werden kann. Die Reagenzien, die nicht mit Metall in Kontakt kommen dürfen, werden mit Kunststoffspritzen und -Kathedern eingefüllt (Abb. 1).

\section{Säulenpassage}

Der eingefrorene Serumüberstand (2-3 Wochen ohne Verlust haltbar) wird nach dem Auftauen sofort mit $\mathrm{K}_{2} \mathrm{CO}_{3}$ auf $\mathrm{pH}$ 5,5 eingestellt. Sobald sich das entstehende Kaliumperchlorat abgesetzt hat, kann der klare Überstand die Säule passieren. Die Durchflußgeschwindigkeit wird durch leichten Druck bei $1 \mathrm{ml}$ pro 5 Min. gehalten.

Sollen auch die gebundenen Katecholamine bestimmt werden, so muß die abtropfende Flüssigkeit aufgefangen werden und nach $10 \mathrm{Min}$. Säurehydrolyse mit Perchlorsäure bei $100^{\circ}$ wie oben angegeben nochmals eine $z$ weite Säule passieren.

\section{Elution}

Die Reste des Serumüberstandes werden mit

$3 \mathrm{~m} l$ Phosphatpuffer und

$1 \mathrm{~m} l$ quartdest. Wasser

aus der Säule entfernt. Die Elution erfolgt dann mit $3 \mathrm{~m} l 1 \mathrm{~N} \mathrm{HCl}$.

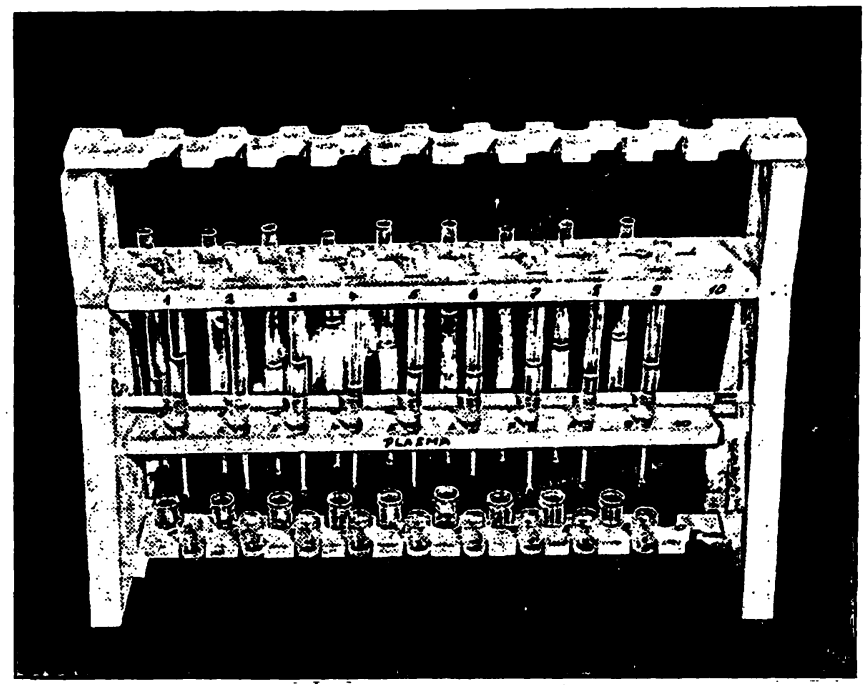

Abb. 2 Elution. Das Eluat wird in $\underset{\text { graduierten Kunststoffbechern aufge- }}{ }$

Die Durchflußgeschwindigkeit darf hierbei nicht erhöht werden (Abb. 2). Die Katecholamine werden bei einem $\mathrm{pH}$ unter 2,3 eluiert. Die fortlaufende $\mathrm{pH}-\mathrm{Kon}$ -

\begin{tabular}{|c|c|c|}
\hline & Meßwert & Leerwert \\
\hline $\begin{array}{l}\text { Phosphatpuffer } \\
\mathrm{K}_{3} \mathrm{Fe}(\mathrm{CN})_{6}\end{array}$ & $\begin{array}{l}50 \mu l \\
30 \mu l\end{array}$ & $\begin{array}{l}50 \mu l \\
30 \mu l\end{array}$ \\
\hline & Nach 3 Min. & \\
\hline $\begin{array}{l}\mathrm{BAL}-\mathrm{Na}_{2} \mathrm{SO}_{3} \\
\mathrm{NaOH} 1 \mathrm{ON}^{-}\end{array}$ & $\begin{array}{r}60 \mu l \\
100 \mu l\end{array}$ & $\overline{100} \mu l$ \\
\hline $\mathrm{BAL}-\mathrm{Na}_{2} \mathrm{SO}_{3}$ & Nach 10 Min. & $60 \mu l$ \\
\hline
\end{tabular}
trolle entfällt durch die Eichung der Säulen (siehe unten).

\section{Nacbweisreaktion}

Das Eluat wird halbiert in Meß- und Leerwert. Beide werden mit $\mathrm{K}_{2} \mathrm{CO}_{3}$ auf $\mathrm{pH}$ 6,5 eingestellt und nach folgendem Schema weiterbehandelt:

\section{Messung}

Die Messung erfolgt $10 \mathrm{Min}$. nach Zugabe der letzten Reagenzlösung. Jeder Wert wird in Quarzküvetten MF 4 bei $395 / 510$ und $335 / 510 \mathrm{~nm}$ Anregung bzw. Emission gemessen. Spaltbreiten: 1,4/0,2 mm. Wegen der notwendigen maximalen Verstärkung muß das Gerät vor jeder Messung mit einem Fluoreszenzstandard geeicht werden.

\section{Auswertung}

\section{Berechnung}

Die Extinktionen der gemessenen Werte werden durch folgende Formel mit den Extinktionen bekannter Eichwerte in Beziehung gesetzt:

$$
\begin{aligned}
& \text { y } N A=\frac{A e_{335} \cdot D_{1}-A e_{395} \cdot D_{2}}{{A e_{335}} \cdot \mathrm{NAe}_{395}-\mathrm{Ae}_{395} \cdot \mathrm{NAe}_{335}} \\
& \text { y } A=\frac{D_{1}-y N A \cdot N A e_{395}}{A e_{305}}
\end{aligned}
$$

Z. klin. Chem. u. klin. Biochem. / 8. Jahrg. 1970 / Heft 1 
$\mathrm{D}_{1}=$ Differenz Meßwert-Leerwert bei 395/510 nm $\mathrm{D}_{2}=$ Differenz Meßwert-Leerwert bei $335 / 510 \mathrm{~nm}$ $\mathrm{Ae}=$ Adrenalineichwert bei angegebener Anregung $\mathrm{NAe}=$ Noradrenalineichwert bei angegebener Anregung.

Die Konzentration in $\mathrm{ng} / \mathrm{m} /$ ergibt sich für Adrenalin aus y $\mathrm{A}$ bzw. für Noradrenalin aus y NA durch Division durch Plasma/2 und Multiplikation mit dem Faktor 10.

\section{Eichwerte}

Eine Standardlösung enthält $1 \mu \mathrm{g} / \mathrm{ml}$ Adrenalin bzw. Noradrenalin in $0,005 \mathrm{~N} \mathrm{HCl}$ und soll jeweils frisch angesetzt werden. Es wird die Nachweisreaktion durchgeführt. Anstelle des $\mathrm{Meß}$ - und Leerwertes tritt das gleiche Volumen 1s HCl. $10 \mu l$ Standard werden einem der beiden Werte zugesetzt, der damit zum Meßwert wird. Die Differenz der Extinktion dieses StandardMeßwertes zum Leerwert wird sinngemäß in obige Gleichung eingesetzt.

\section{Eicbung der Säulen}

Durch die Eichung wird ermittelt, wann eine vollständige Adsorption der Katecholamine an den Kationenaustauscher stattfindet und das Volumen des Eluates am geringsten ist. Dazu werden je zwei Säulen mit 40, 50, 60 und $70 \mathrm{mg}$ Austauscherharz gefüllt. Nach dem Waschen erfolgt die Passage einer Testlösung, die pro Säule aus $1 \mathrm{ml}$ quartdest. Wasser
$+100 \mu l$ Phosphatpuffer $+10 \mu l$ Adrenalin $+10 \mu l$ Noradrenalin-Standard besteht. Die abtropfende Testlösung wird aufgefangen, halbiert und als Meß- bzw. Leerwert gemessen. Es genügt, die Säulen vor der nun folgenden Elution nur mit $2 \mathrm{ml}$ quartdest. Wasser zu waschen. Das abtropfende Eluat wird in $0,3 \mathrm{ml}$ Portionen aufgefangen, neutralisiert und als Meßwert gemessen. Die Messungen brauchen nur bei 395/510 nm erfolgen, da die quantitative Differenzierung unnötig ist.

Beispiel:

\begin{tabular}{|c|c|c|c|c|c|c|c|c|}
\hline \multirow[t]{2}{*}{$\begin{array}{c}\text { Säule } \\
\text { Nr. }\end{array}$} & \multirow[t]{2}{*}{$\underset{\mathrm{mg}}{\text { Harz }}$} & \multirow{2}{*}{\multicolumn{2}{|c|}{$\begin{array}{c}\text { Abgetropfte } \\
\text { Testlösung } \\
\text { Meßw. Leerw. }\end{array}$}} & \multicolumn{5}{|c|}{$0,3 \mathrm{ml}$-Portion $\mathrm{Nr}$. } \\
\hline & & & & 1 & 2 & 3 & 4 & 5 \\
\hline $\begin{array}{l}1 \\
2 \\
3 \\
4\end{array}$ & $\begin{array}{l}70 \\
60 \\
50 \\
40\end{array}$ & $\begin{array}{l}0,5 \\
0,5 \\
0,5 \\
5,0\end{array}$ & $\begin{array}{l}0,5 \\
0,5 \\
0,5 \\
0,5\end{array}$ & $\begin{array}{r}0,5 \\
0,5 \\
0,5 \\
40,0\end{array}$ & $\begin{array}{r}2,0 \\
6,0 \\
33,0 \\
5,0\end{array}$ & $\begin{array}{r}10,0 \\
21,0 \\
17,0 \\
0,5\end{array}$ & $\begin{array}{r}20,0 \\
21,0 \\
1,0 \\
0,5\end{array}$ & $\begin{array}{r}20,0 \\
2,0 \\
0,5 \\
0,5\end{array}$ \\
\hline
\end{tabular}

Die Säule Nr. 3 ist in diesem Beispiel mit der optimalen Menge des Kationenaustauschers gefüllt. Die ersten $0,3 \mathrm{ml}$ werden folglich bei der Elution verworfen, die folgenden $0,6 \mathrm{~m} /$ als Eluat aufgefangen.

\section{Empfindlicbleeit der Methode}

Die Wiederfindung beträgt bei 30 Bestimmungen und einer Katecholaminkonzentration von $1 \mu \mathrm{g} / \mathrm{ml}$ für Adrenalin $70,5 \pm 3,6 \%$ und für Noradrenalin $63,5 \pm 3,8 \%$.

\section{Literatur}

1. Häggendal, J., Pharmacol. Rev. 18, 325 (1966). - 2. Bertler, A., A. Carlsson und E. Rosengren, Acta physiol. Scand. 44, 273 (1958). - 3. HäGGENDAL, J., Acta physiol. Scand. 59, 242 (1963). - 4. Lund, A., Acta pharmac. tox. Khvn. 5, 121 (1949). 5. Euler, v. U. S., Pharmacol. Rev. 11, 262 (1959). - 6. WeIL-
Malherbe, H. und A. D. Bone, Biochem. J. 67, 65 (1957). 7. Cohen, G. und M. Goldenberg, J. Neurochem. 2, 58 (1957). 8. Price, H. L. und M. L. Price, J. Laborat. Clin. Med. S. Louis, 50,769 (1957).
Dr. G. Hollmann

Kinderchirurgische Klinik der Kinderklinik der Universität München

8 München 15

Lindwurmstr. 4 